\title{
High toughness fibrillating metal-elastomer interfaces: on the role of discrete fibrils within the fracture process zone
}

Citation for published version (APA):

Vossen, B. G., van der Sluis, O., Schreurs, P. J. G., \& Geers, M. G. D. (2016). High toughness fibrillating metalelastomer interfaces: on the role of discrete fibrils within the fracture process zone. Engineering Fracture Mechanics, 164, 93-105. https://doi.org/10.1016/j.engfracmech.2016.05.019

DOI:

10.1016/j.engfracmech.2016.05.019

Document status and date:

Published: 21/08/2016

\section{Document Version:}

Accepted manuscript including changes made at the peer-review stage

\section{Please check the document version of this publication:}

- A submitted manuscript is the version of the article upon submission and before peer-review. There can be important differences between the submitted version and the official published version of record. People interested in the research are advised to contact the author for the final version of the publication, or visit the $\mathrm{DOI}$ to the publisher's website.

- The final author version and the galley proof are versions of the publication after peer review.

- The final published version features the final layout of the paper including the volume, issue and page numbers.

Link to publication

\section{General rights}

Copyright and moral rights for the publications made accessible in the public portal are retained by the authors and/or other copyright owners and it is a condition of accessing publications that users recognise and abide by the legal requirements associated with these rights.

- Users may download and print one copy of any publication from the public portal for the purpose of private study or research.

- You may not further distribute the material or use it for any profit-making activity or commercial gain

- You may freely distribute the URL identifying the publication in the public portal.

If the publication is distributed under the terms of Article 25fa of the Dutch Copyright Act, indicated by the "Taverne" license above, please follow below link for the End User Agreement:

www.tue.nl/taverne

Take down policy

If you believe that this document breaches copyright please contact us at:

openaccess@tue.nl

providing details and we will investigate your claim. 


\title{
High toughness fibrillating metal-elastomer interfaces: on the role of discrete fibrils within the fracture process zone
}

\author{
B.G. Vossen ${ }^{\mathrm{a}}$, O. van der Sluiss ${ }^{\mathrm{a}, \mathrm{b}, *}$, P.J.G. Schreurs ${ }^{\mathrm{a}}$, M.G.D. Geers ${ }^{\mathrm{a}}$ \\ ${ }^{a}$ Department of Mechanical Engineering, Eindhoven University of Technology \\ P.O. Box 513, 5600 MB Eindhoven, The Netherlands \\ ${ }^{b}$ Philips Research Laboratories, High Tech Campus 34, 5656 AE Eindhoven, The Netherlands
}

\begin{abstract}
Fibrillating metal-elastomer interfacial systems, typically used in stretchable electronics applications, can exhibit remarkably high values for the interface fracture toughness. Consequently, a huge gap exists between the low adhesion energy at the microscopic scale and the measured macroscopic work of separation. This contribution aims to close this energy gap by unravelling the underlying dissipative mechanisms through a multi-scale approach. The first scale transition was established in earlier work, and concerned the formation and deformation of a single fibril at the copper-rubber interface up to failure. It was shown that the obtained work of separation was significantly larger than the small-scale interface adhesion, yet a decade too small with respect to the experimental values. In order to close the energy gap, in this contribution, the second scale transition is achieved by considering a finite number of elongating discrete hyperelastic fibrils within the fracture process zone. It is shown that the dynamic release of the stored elastic energy by fibril fracture that results from the spatial discreteness of multiple fibrils, the interaction with the adjacent deforming bulk elastomer material and the highly nonlinear behavior of the elastomer provides an explanation for the high work of separation values. In addition, an intrinsic shortcoming of cohesive zone formulations at the macroscopic scale is revealed. The results provide a mechanistic understanding of the physics involved with interface delamination through fibrillation in metal-elastomer interfaces.
\end{abstract}

Keywords: fibrillation, stretchable electronics, delamination, fracture process zone, work-of-separation, PDMS

\section{Introduction}

Stretchable electronics are nowadays finding applications in a variety of novel products, ranging from electronic textiles to biomedical applications such as epidermal electronic systems, stretchable optoelectronics and advanced surgical tools (Coosemans et al., 2006; Hsu et al., 2009, 2010; Khang et al., 2006; Kim et al., 2011a,b; Li et al., 2005; Rogers et al., 2010; Song et al., 2008). These applications benefit from the increased design freedom and comfort offered by devices that are deformable, as opposed to the conventional stiff semiconductor microelectronics. Stretchable electronics typically consist of semiconductor islands, embedded in highly compliant substrates, often made of a rubber material. The electrical connectivity is achieved through thin metal interconnect lines. The mechanical integrity of the conductor lines is one of the main reliability issues for stretchable electronics, since fracture of the conductor lines, as discussed by Gonzalez et al. (2008) and Li and Suo (2007), will lead to immediate failure of the product due to the loss of electrical functionality. It has been observed that the reliability of the product is

\footnotetext{
${ }^{*}$ Corresponding author. Tel.: +31 402472843

Email address: o.v.d.sluis@tue.nl (O. van der Sluis)
} 
mainly dictated by the integrity of the interface between the conductor lines and the substrate. Lu et al. (2007) discuss that, as long as the interface is intact, the strain in the conductor lines is delocalized by the substrate. Delamination may act as a precursor to necking of the metal and subsequently to fracture, thus leading to electrical failure. Furthermore, Hsu et al. (2010) point out that delamination may cause exposure of the interconnect to the environment, possibly leading to premature metal failure due to the effect of for example humidity or salt corrosion. Clearly, understanding the interface behavior is of key importance to engineer reliable products.

Dedicated experiments on these particular metal-elastomer systems, Cu-poly(dimethylsiloxane) (PDMS) rubber, provide better understanding of the actual mechanisms in the fracture process zone. It has been observed that the interface delaminates through rubber fibrillation, a process that involves the formation, elongation and rupture of rubber fibrils, as shown in Fig. 1. From these peel experiments, notably high values for the work of separation have been reported (Hoefnagels et al., 2010; Van der Sluis et al., 2011), $G_{c}>1 \mathrm{~kJ} / \mathrm{m}^{2}$. This contribution aims to unravel the huge gap between the microscopic adhesion energy of a copper-rubber system and its measured macroscopic work of separation which is of key importance for interfacial engineering of stretchable electronics.

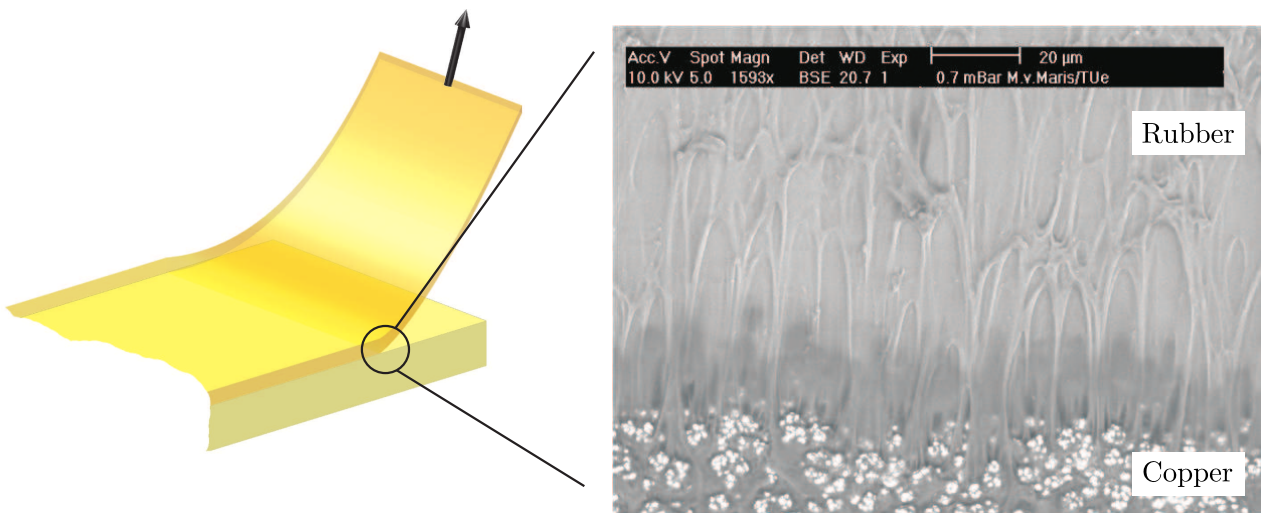

Figure 1: In situ scanning electron microscopy observation of PDMS fibrillation during a Cu-PDMS peel test.

A vast body of literature on fibrillar structures exists, see for example the review paper by Jagota and Hui (2011). Here, fibrillar structures are studied that are in direct contact with the substrate, resulting in the loss of elastically stored energy in the fibril upon detachment, which is considered to be the main dissipative mechanism in these systems. Jagota and Bennison (2002) used such a system to study adhesion enhancement induced by a fibrillar structure. Later, Glassmaker et al. (2004); Hui et al. (2004) studied the contact and adhesion between a PDMS fibrillar structure and a rigid substrate, mainly focusing on the relation between the pull-off stress and the properties of the fibrils. The adhesion enhancement has been studied in more detail by e.g. Glassmaker et al. (2005); Shen and Soh (2008); Tang et al. (2005). Typically, the energy loss is calculated analytically assuming linear elastic material behavior, which enables to directly establish the amount of energy in a fibril based on a given (simple) geometry loaded 
up to a certain stress level. More recently, Guidoni et al. (2010) used finite strain elasticity to determine the energy contribution of the fibrils. The aforementioned studies do not account for the energy storage and loss in the bulk layer interconnecting the fibrillar structure. Although Glassmaker et al. (2005) observed a discrepancy between their theoretical model and experimental data, which was attributed to the viscoelastic energy loss in the bulk region outside the fibrils, they did not explore this effect further, and their analysis mainly focused on the role of energy loss within the fibril. Furthermore, following the work of Noderer et al. (2007), the effect of the deformation of the backing layer was accounted for in a small strain, linear elasticity setting by Guidoni et al. (2010) in their work on contact compliance of fibrillar structures. Long et al. (2008) used a continuous elastic foundation to model the discrete fibrils and used linear elasticity theory to relate the pull-off force of a fibrillar array to the thickness of the surrounding bulk layer. However, the contribution of the deformation of the bulk layer to the total energy loss was not analyzed, nor quantified, in these studies. More recently, Zhao and co-workers (Yuk et al., 2016; Zhao, 2014) reported very high work of separation values of intrinsically tough hydrogels which was achieved by chemically anchoring the hydrogel on a solid substrate. The anchoring mechanism resulted in large deformations in the hydrogel in which a significant amount of energy was dissipated by reversible crosslinking and chain scission and contributed to the interfacial toughness.

Fibrillation micro-mechanics has received considerable attention, especially for viscoelastic pressuresensitive adhesives (PSAs), as discussed in Creton (2003). Zosel (1998) and Creton and co-workers (Brown et al., 2002; Lakrout et al., 1999; Shull and Creton, 2004) used tack tests to gain insight in the parameters that control the fibrillation process. A rigid cylindrical punch was brought into contact with a layer of PSA, and after establishing good contact the punch was retracted. Their experiments show that the fibrillation process consists of several phases. The first phase is homogeneous deformation of the film, after which cavities nucleate at the interface between the two materials. Increasing the loading leads to the growth of these cavities. Consequently, a fibrillar structure is formed. The interface fails by either interfacial cavitation at low loading rates, or by a bulk cavitation at higher loading rates, both followed by interfacial detachment of the fibrils (Brown et al., 2002). The essential differences between PSAs and our material system are: (i) PSA fibril material is viscoelastic while PDMS is nearly hyperelastic; (ii) PSA fibril lengths are in the millimeter range while the PDMS fibrils are only tens of micrometers long. As a result, the relative contribution of the fibril elongation to the work of separation for both systems appears to be rather different: for PSAs, fibril mechanics is essential due to the significant energy dissipation in these largely elongated viscoelastic fibrils, while for PDMS, fibril elongation constitutes only a minor contribution to the work of separation.

In earlier work, the micro-mechanical contributions of the fibril to the macroscopic work of separation were investigated for a $\mathrm{Cu}-\mathrm{PDMS}$ system. To this end, a finite element multi-scale interface framework 
was used, in which the macro-scale interface description was extracted from a single fibril micro-model including fibril debonding (Vossen et al., 2014). Mode I loading was applied to a nucleated fibril, and the response was simulated up to the moment of fibril failure. The highly nonlinear hyperelastic material response at large strains was taken into account by an Ogden model and the results were experimentally assessed using a single fibril experiment (Vossen et al., 2015). The employed fibril model clearly relies on recently reported experimental results in Neggers et al. (2015), demonstrating the following microscopic characteristics: (i) fibrils are mostly attached to the surface roughness valleys before failure; (ii) in spite of the large deformation, inelasticity of the PDMS at fibril scale is negligible; (iii) fibril fracture occurs. The model results suggested that the dissipated energy mainly consists of elastically stored energy that is lost through dynamical release upon unstable fibril failure. Even though the resulting work of separation was almost two orders of magnitude larger than typical values for the micro-scale adhesion energy, the single fibril micro-mechanics model significantly underestimated the work of separation and thus, could not fully explain the reported values of $\mathrm{Cu}-\mathrm{PDMS}$ interfaces.

This paper aims to close the large energy gap in the resulting work-of-separation, compared to previously reported results. To achieve this, a discrete multi-fibril model is here proposed that describes a finite number of elongating fibrils within the fracture process zone, in order to analyze the mutual interaction between the fibrils, the interaction with the adjacent bulk PDMS and the role of discreteness of the fibrils. Upon loading, fibril failure occurs instantaneously once the critical load is reached. First, using a simplified mode I analysis in which all fibrils fail simultaneously, it is demonstrated that the combination of discrete spacing with unstable failure of the fibrils and the nonlinear PDMS material behavior are essential for the energy loss in the bulk. The relation between discreteness (by varying the fibril spacing at constant fibril density) and work-of-separation is quantified. Next, while preserving the discrete character of the system, the energy loss in the bulk is determined for a more realistic condition: sequential fibril failure. Due to the less confined geometry, a much larger volume of material deforms. Upon fibril failure, a substantial amount of energy in this volume is released dynamically, leading to a significantly larger macroscopic work-of-separation compared to the simultaneous failure case. Interestingly, in the continuum limit this energy is not recovered and no longer present in the bulk layers, which illustrates an intrinsic limitation of continuum cohesive zone models when applied to highly compliant systems for which the discrete character is essential. Finally, by studying the effect of the fibril strength on the work-of-separation, a quantitative relation between the height of the elastomer lift-off geometry (i.e. the fracture process zone) and the calculated work-of-separation has been established. The results provide a mechanistic understanding of the physics involved with interface delamination through fibrillation in metal-elastomer interfaces and an explanation for the high work-of-separation values. 


\section{Model overview}

\subsection{Geometry}

The model geometry is schematically shown in Fig. 2. For simplicity, a plane strain geometry is adopted, with out-of-plane thickness $t$, taken as unity. The height of the bulk layer $H=1 \mathrm{~mm}$, which corresponds to the layer height used in the experiments reported in Van der Sluis et al. (2011). The bulk layer is attached to the substrate through a finite number of fibrils. The substrate roughness may be a limiting factor for establishing adhesion and for the interfacial properties in general (Hui et al., 2005, 2007; Porwal and Hui, 2008; Tang et al., 2005). Furthermore, when the fibrils are long and thin, they may stick laterally before adhering to the substrate (Glassmaker et al., 2004; Hui et al., 2002; Persson, 2003; Sitti and Fearing, 2003), thereby reducing the adhesion area and possibly limiting the interface properties. However, during Cu-PDMS interface delamination, fibrils are drawn from the bulk PDMS material instead of being pre-existent. The fact that the fibrils are therefore always in tension makes lateral sticking unlikely. Even if lateral sticking would occur during fibril drawing, this would not reduce the adhesion properties, since adhesion is established prior to sticking. Furthermore, the roughness does not limit the adhesion area, since the rubber is cast onto the copper in an uncured state. Assuming perfect wetting, the casting and curing enforces the shape of the rubber to match the copper geometry quite exactly. Hence, the assumption of initially perfect adhesion is appropriate for the considered samples. Cavitation, which is the initial phase of fibrillation, is not considered in the model. Even though this is a key mechanism for the initiation of the fibrillation process (Lakrout et al., 1999; Zosel, 1998), it is not important in terms of dissipation. For a fully bonded material layer, the stress at the top of the roughness peaks will rapidly increase upon loading, leading to low energy storage before cavity formation, as cavitation is a stress-driven mechanism.

Since the stiffness of the Cu-substrate is much higher than the PDMS stiffness, the substrate is considered to be rigid. The fibril dimensions are: height $H_{f}=10 \mu \mathrm{m}$, and width $w$, while the spacing between fibrils is denoted by $h$. The influence of $w$ on the dissipated energy will be studied in detail. For comparison purposes, discrete structures with $h=w$ and thus having an identical fibril density, are used

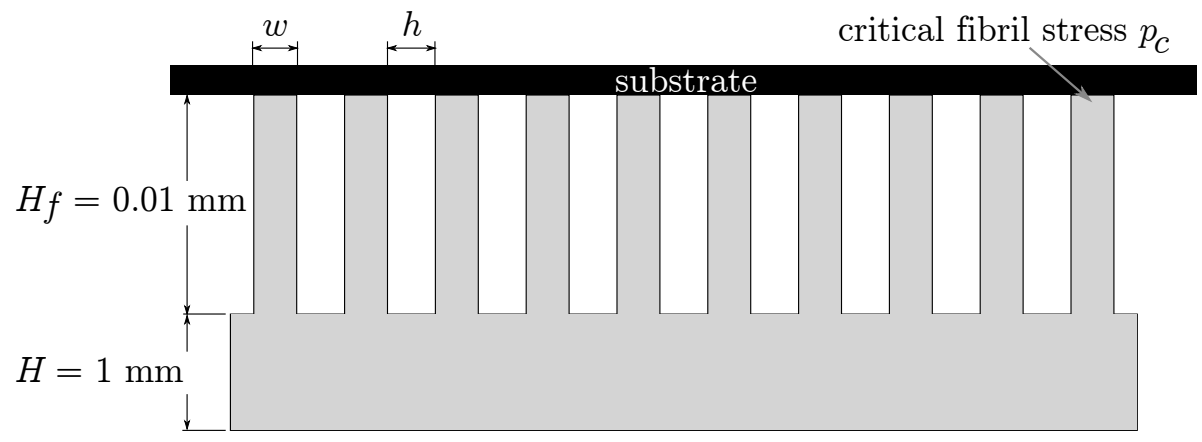

Figure 2: Overview of the fibrillar geometry (not to scale). 
throughout this paper.

\subsection{Material properties}

The rubber material is modelled using an Ogden formulation, which captures the strain hardening typically observed for rubbers at large strains. The strain energy density function $W$ (per unit undeformed volume) is given by (Maas et al., 2012)

$$
W=\frac{1}{2} \kappa(J-1)^{2}+\sum_{k=1}^{N} \frac{c_{k}}{m_{k}^{2}}\left(\lambda_{1}^{m_{k}}+\lambda_{2}^{m_{k}}+\lambda_{3}^{m_{k}}-3-m_{k} \ln J\right),
$$

where $\lambda_{i}$ are the principal stretches, $J=\operatorname{det}(\mathbf{F})$ is the volume change ratio, $\mathbf{F}$ the deformation gradient tensor, and $c_{k}$ and $m_{k}$ are material parameters. $N$ denotes the number of terms; $N=2$ is used for all simulations in this paper. $\kappa$ is the bulk modulus, which is essentially a penalty parameter to enforce the near incompressibility of the material. Its value is taken such that the initial Poisson's ratio equals $\nu=0.499$. The Cauchy stress tensor $\boldsymbol{\sigma}$ can be written as

$$
\boldsymbol{\sigma}=\sum_{i=1}^{3} \sigma_{i} \vec{n}_{i} \vec{n}_{i}
$$

where $\vec{n}_{i}$ are the eigenvectors of the Finger tensor $\mathbf{B}$ and the principal stresses $\sigma_{i}$ can be derived from the strain energy density function, resulting in

$$
\sigma_{i}=\kappa(J-1)+\sum_{k=1}^{N} \frac{1}{J} \frac{c_{k}}{m_{k}}\left(\lambda_{i}^{m_{k}}-1\right) .
$$

The material parameters are listed in Table 1, which are representative for Sylgard $186^{\circledR}$ (Dow Corning), a commonly applied PDMS material in stretchable electronics, and in fact used in the peel test samples reported in Hoefnagels et al. (2010) and Neggers et al. (2015).

Table 1: PDMS material model parameters used in the simulations
\begin{tabular}{cccccc}
\hline$m_{1}[-]$ & $c_{1}[\mathrm{MPa}]$ & $m_{2}[-]$ & $c_{2}[\mathrm{MPa}]$ & $\kappa[\mathrm{MPa}]$ \\
\hline 6.8 & 0.00783 & 0.799 & 0.682 & 172.14 \\
\hline
\end{tabular}

The governing failure mechanisms of the fibrillar structure at the micro-scale are fibril debonding and fibril fracture. For the considered Cu-PDMS material system, it was shown that the actual debonding mechanism is rather insignificant in terms of macroscopic work-of-separation values (Vossen et al., 2014). Without compromising the physics of the underlying dissipative processes, the failure of the fibrillar structure is approximated by the following failure criterion: fibrils are assumed to fail instantaneously once the magnitude of the average first Piola-Kirchhoff stress $p$ on the fibril's top end reaches the critical 
stress $p_{c}$. A value of $p_{c}=2 \mathrm{MPa}$ is adopted, which is motivated by the results of a previous micromechanical analysis (Vossen et al., 2015). The influence of different values on the results will be shown in Section 5.

\subsection{Loading conditions}

Two distinctive loading cases are considered. The first case is simultaneous failure of all fibrils under mode I loading. This loading case is often considered in the literature on fibrillar structures, where it is named equal load sharing (ELS) (Hui et al., 2004). It is remarked that this loading condition reflects a highly idealized situation, and is thus of limited value for realistic loading cases, yet, it does enable a systematic study of the different contributions to the work-of-separation. This loading mode can be fully analyzed by means of a single fibril, with appropriate boundary conditions, as shown in Fig. 3a. This loading case highly confines the bulk material. The effect of the fibril width $w$ on the energy loss in both the fibril and the bulk will be analyzed. The fibril is elongated in vertical direction until the failure criterion is reached.

The second case is sequential failure of the fibrils. In a peel test, the fibrils do not fail simultaneously, rather they fail sequentially as the peel front progresses through the interface. This implies that the material is much less confined compared to the mode I simultaneous failure case, since material can be drawn to the peel front from the adjacent bulk material. The loading conditions are shown schematically in Fig. 3b. Both mode I and mixed mode sequential failure of the fibrils will be analyzed. To assure steady state peeling conditions, the crack front is placed in the center of the geometry. The sequential case aims to reflect the peel loading in a simplified way, in order to limit the analysis to the essentials in terms of identification of dissipative mechanisms during peeling. Numerical analysis of the actual peel test, consisting of the Cu-film and the PDMS material indicates that the energy storage in the copper film, due to plasticity, is very limited, and thus, the film can safely be replaced by a set of boundary conditions directly applied on the fibril ends. The completely failed fibrils do not carry any load and are

(a)

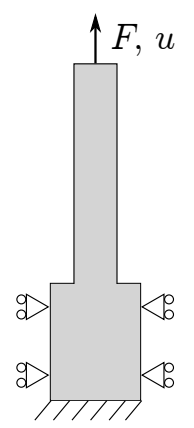

(b)

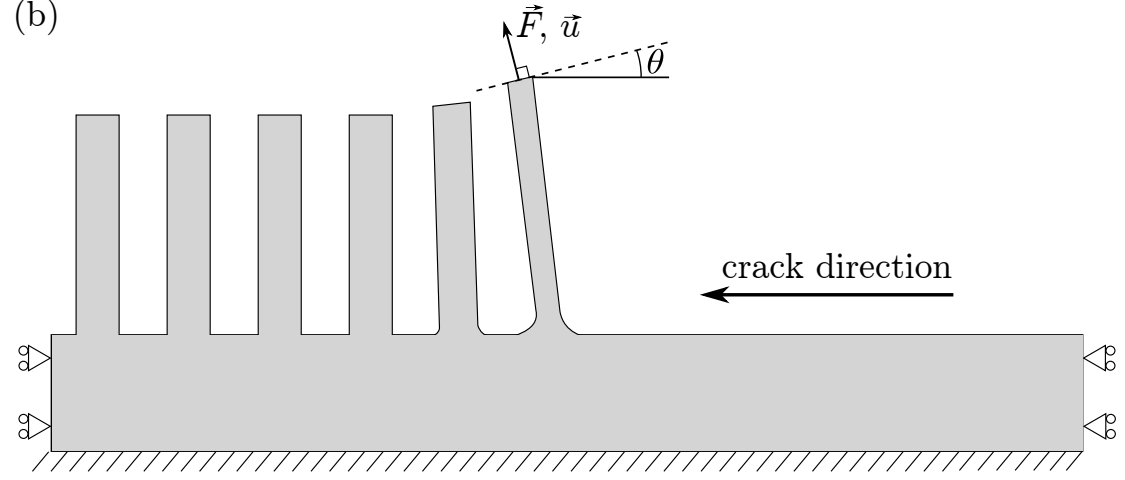

Figure 3: (a) Simultaneous failure of all fibrils is simulated using a single fibril; (b) For sequential failure, load is applied to the fibrils at a loading angle $\theta$. The crack propagates from right to left whereas the already failed fibrils are not shown. 
therefore omitted from the model (the crack front travels from right to left in Fig. 3b). Due to the loading conditions, fibrils stretch at angle $\theta$ until the failure criterion is reached. For the mode I case, sequential failure is realized by applying the load under a negligibly small angle $\theta=1^{\circ}$. The substrate/fibril interaction is captured through appropriate boundary conditions that are directly applied to the fibrils, enabling free contraction and rotation of the fibril extremity. Krishnan and Hui (2008) showed that the type of boundary conditions applied at the fibril extremity has a limited influence on the resulting response under mode I loading. Moreover, since this paper focuses on the effect of the discrete fibrils on the bulk behavior, whereby boundary conditions are applied at the fibril extremity, i.e. away from the bulk, their influence on the final results are expected to be negligible.

The finite element simulations are performed within a geometric nonlinear framework using four node bilinear quadrilateral elements. To avoid volumetric locking typically associated with (nearly) incompressible materials, selective reduced integration is used. Fibril failure is accomplished by releasing the corresponding displacement constraints at the top end of the fibril.

\section{Simultaneous fibril failure}

An example of the deforming geometry is shown in Fig. 4, in which the fibril width $w$ equals $0.01 \mathrm{~mm}$ and the colors indicate the strain energy density (SED) $W\left[\mathrm{~N} / \mathrm{mm}^{2}\right]$. Upon reaching the failure criterion, the total SED is determined and can be decomposed into fibril and bulk contributions. Both terms constitute the total amount of energy that is stored throughout the loading process, and subsequently released upon unstable failure (i.e. dissipated dynamically). If the local failure events would be stable and all materials would be elastic, the energy would be recovered gradually and the only term in the workof-separation would be the intrinsic adhesion energy. In the present analysis, for the considered failure criterion, the local failure is always unstable, consistent with previous results (Vossen et al., 2014) and, consequently, all the elastically stored energy contributes to the work-of-separation upon fibril failure.

\subsection{Analytical approximations}

First, assuming simple stress and deformation states, analytical approximations and scaling laws for the energy loss in both the bulk and the fibril are established.

\subsubsection{Fibril energy loss}

The fibril is assumed to be in a plane strain tension state, as depicted in Fig. 5a. This assumption is similar to Glassmaker et al. (2005); Guidoni et al. (2010); Shen and Soh (2008); Tang et al. (2005), yet the fact that a nearly incompressible finite strain constitutive model is used here instead of a fully incompressible one, necessitates solving the equations iteratively. The transverse stress $\sigma_{x}$ is equal to 


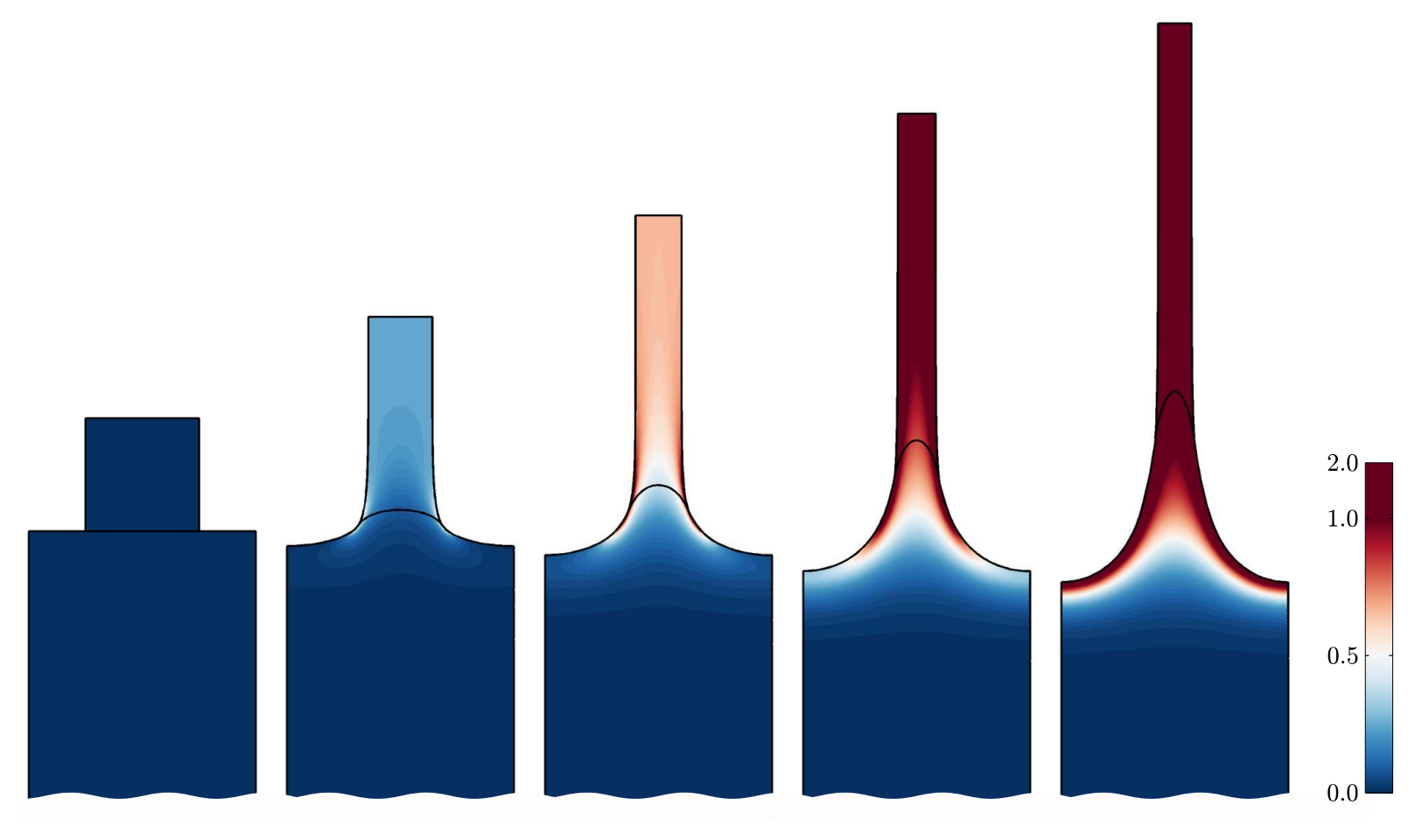

Figure 4: Several loading stages for the simultaneous failure case (only part of the model is shown), in which the fibril width $w=0.01 \mathrm{~mm}$ and the colors indicate the strain energy density $W\left[\mathrm{~N} / \mathrm{mm}^{2}\right]$. The evolving solid black line is included for visualization purpose.

zero, and the tensile Cauchy stress $\sigma_{y}$ is equal to $p_{c} / \lambda_{x}$ (recall that $p_{c}$ is the first Piola-Kirchhoff failure stress), leading to

$$
\begin{aligned}
\sigma_{x} & =\kappa(J-1)+\sum_{k=1}^{N} \frac{1}{J} \frac{c_{k}}{m_{k}}\left(\lambda_{x}^{m_{k}}-1\right)=0, \\
\sigma_{y} & =\kappa(J-1)+\sum_{k=1}^{N} \frac{1}{J} \frac{c_{k}}{m_{k}}\left(\lambda_{y}^{m_{k}}-1\right)=p_{c} / \lambda_{x} .
\end{aligned}
$$

Solving the coupled equations (4) yields the principal stretches $\lambda_{x}$ and $\lambda_{y}$. Substituting these values in the expression for the strain energy density $W$, equation (1), allows to calculate the SED in the fibril, $W_{f}\left(\lambda_{x}, \lambda_{y}\right)$. Note that the obtained value for $W_{f}$ does not depend on any geometrical parameters. The corresponding work-of-separation is simply the amount of released energy (strain energy density multiplied by the undeformed volume) divided by the total interfacial area (recall the unit thickness, and $w=h)$,

$$
\Gamma_{f}=W_{f}\left(\lambda_{x}, \lambda_{y}\right) \frac{H_{f} w}{w+h}=W_{f}\left(\lambda_{x}, \lambda_{y}\right) \frac{H_{f}}{2} .
$$

\subsubsection{Bulk energy loss}

At a sufficiently large distance from the fibrils, the stress state in the layer is uniform, where the Cauchy stress in the loading direction is simply $p_{c} / 2$ (since $w=h$ and $\lambda_{x}=1$ ), shown in Fig. 5b. To 

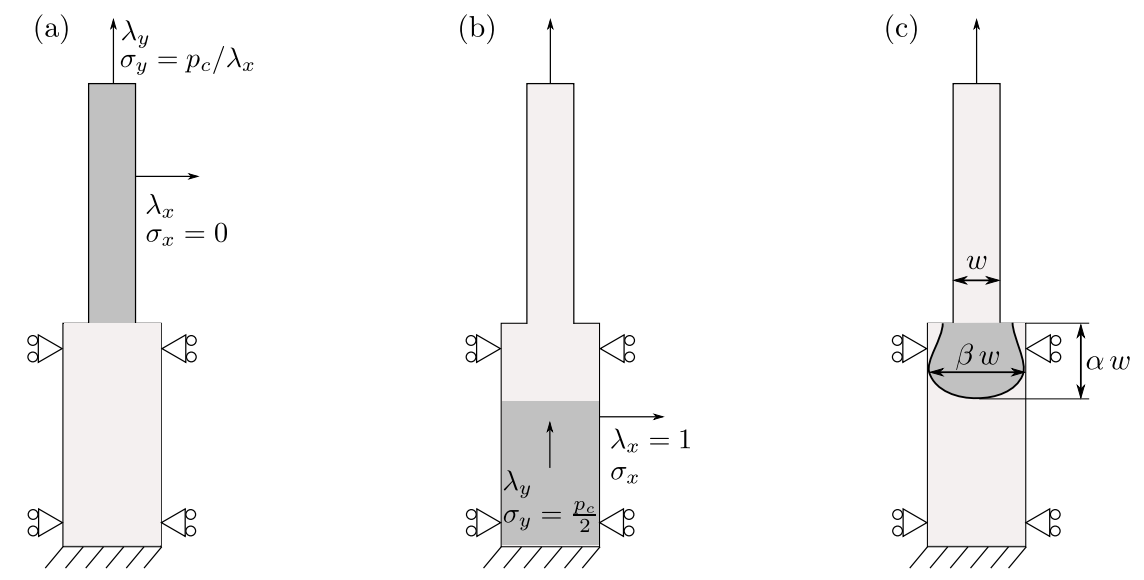

Figure 5: (a) Plane strain tension state in the fibril. (b) At a sufficiently large distance from the fibril, the bulk layer deforms homogeneously. (c) The bulk volume that is affected by the fibril scales with $w^{2} ; \alpha$ and $\beta$ are scaling constants to indicate the size of the affected bulk volume.

extract the stored energy in the bulk material, the following equation is solved

$$
\sigma_{y}=\kappa\left(\lambda_{y}-1\right)+\sum_{k=1}^{N} \frac{1}{J} \frac{c_{k}}{m_{k}}\left(\lambda_{y}^{m_{k}}-1\right)=p_{c} / 2
$$

Solving this equation for $\lambda_{y}$ and inserting the result in the strain energy density $W$, equation (1), allows to calculate the SED in the bulk material, $W_{b c}\left(\lambda_{y}\right)$ for the highly confined and continuous bulk layer. If the entire bulk layer would be in a homogeneous stress state, the contribution to the work-of-separation is simply a constant, given by

$$
\Gamma_{b c}=W_{b c}\left(\lambda_{y}\right) \frac{H(w+h)}{w+h}=W_{b c}\left(\lambda_{y}\right) H .
$$

However, as illustrated in Fig. 4, upon approaching the bulk-fibril boundary, the deformation in the bulk layer becomes clearly non-uniform, which is schematically depicted in Fig. 5c. The affected bulk volume now scales with $(\alpha w) \cdot(\beta w)$, where $\alpha$ and $\beta$ are scaling constants which indicate the size of the affected bulk volume and are introduced only for the sake of argument (ie. their actual value is not important here). The relevant length scale for the bulk volume that is affected by the fibrils is the fibril width $w$, while the fibril length does not significantly affect the bulk deformation. Consequently, the amount of stored energy in the bulk layer near the fibril-bulk boundary, $G_{b u l k}$, scales with $w^{2}$. Therefore, the contribution of the deformation in the bulk material near the bulk-fibril boundary to the work-ofseparation can be formulated as follows

$$
\Gamma_{b f}=\frac{G_{b u l k}}{w+h} \propto \frac{w^{2}}{2 w}=\frac{w}{2}
$$




\subsection{Numerical results}

Fig. 6a shows both the numerical and analytical values for the work-of-separation due to fibril stretching only, $\Gamma_{f}$. Clearly, this contribution is adequately described by equation (5) as the numerical simulations confirm that changing the fibril width $w$ at constant fibril density does not influence the value of $\Gamma_{f}$. Note that these conclusions might not be valid upon further increasing the aspect ratio $w / H_{f}$. Fig. $6 \mathrm{~b}$ depicts the work-of-separation resulting from energy loss in the bulk as a function of the fibril width $w$. Two regions can be recognized: for thin fibrils, the numerical results correspond to equation (7) while for thicker fibrils, the simulation results converge to equation (8). For small fibril widths, the deforming bulk volume is small, and the main contribution to the work-of-separation originates from the homogeneously deforming bulk layer, c.f. equation (7). For large fibril widths, the deforming bulk volume is large, and the energy lost in the deformed bulk volume near the fibrils constitutes the major contribution to the work-of-separation, c.f. equation (8).

These results suggest that the absolute value of the spacing between the fibrils is important for the energy loss in the bulk (recall that for all simulations $w=h$ ). In fact, reducing the spacing between the fibrils, the energy loss in the bulk material due to the fibrils, $\Gamma_{b f}$, reduces to zero. This would represent the continuum limit in which all fibrils are infinitesimal in width. However, the analysis shows that, for sufficiently separated fibrils, the energy loss in the bulk due to the fibrils might be significantly larger than the homogeneous component. Therefore, it is essential to properly account for the discrete character of the system.

Equation 5 indicates that the fibril energy loss scales linearly with the fibril height $H_{f}$, whereas the bulk energy loss is unaffected by the fibril height. Consequently, changing the initial fibril height will change the ratio between fibril and bulk energy loss. This clearly illustrates that the relative contribution of each identified dissipative mechanism to the work-of-separation depends on the geometrical parameters.
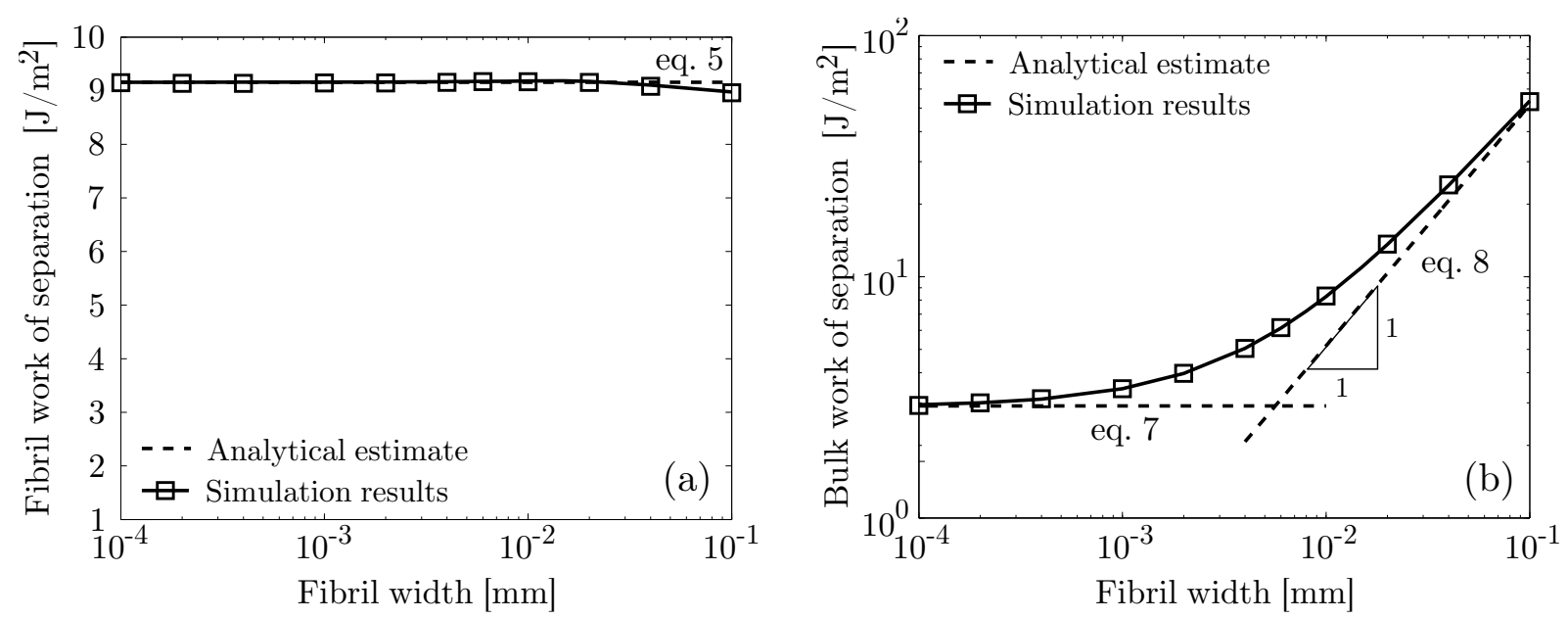

Figure 6: Work-of-separation for simultaneous fibril failure from (a) the fibril $\Gamma_{f}$ and (b) the bulk $\Gamma_{b c}+\Gamma_{b f}$. The dashed lines represent the analytical estimates, the solid lines are the numerical results, where the squares indicate the data points. 

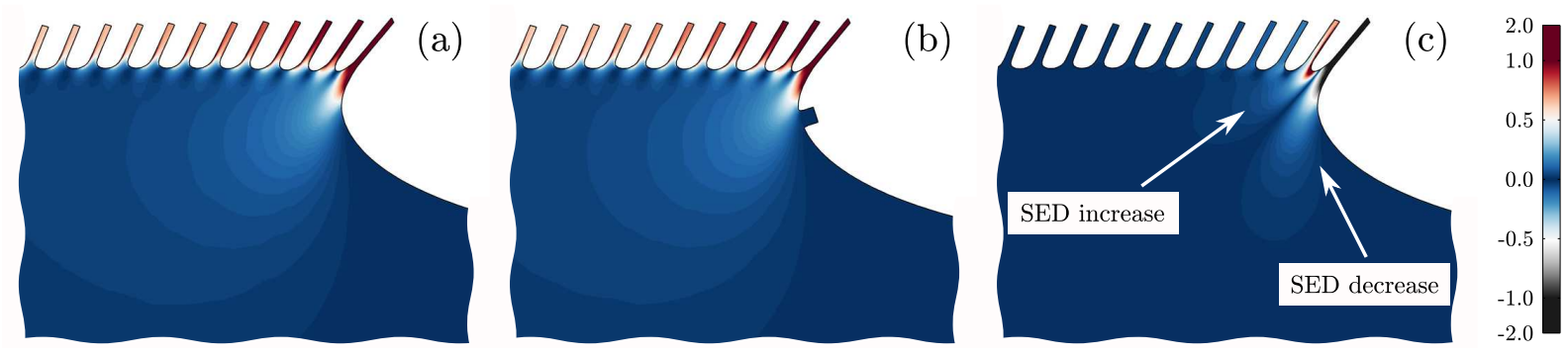

Figure 7: Sequential failure of the fibrillar interface for remote mode I loading $(w=0.01 \mathrm{~mm})$. The colors indicate the strain energy density $\left[\mathrm{N} / \mathrm{mm}^{2}\right]$; (a) The fibril reaches the failure criterion. (b) The deformed geometry after instantaneous failure of the critical fibril. (c) difference in SED projected on the geometry before failure.

For instance, Fig. 6a and Fig. 6b show that the bulk energy loss becomes the dominant term for fibril widths larger than approximately $w=0.01 \mathrm{~mm}, \Gamma_{b f}>\Gamma_{b c}+\Gamma_{f}$. In addition to the geometry, for a bimaterial system, the mismatch in material properties also influences the relative contribution of both terms. Clearly, also in this case the geometrical parameters matter. When the stiffness of the fibril is much larger than the bulk stiffness, most of the energy storage will take place in the bulk and it needs to be properly accounted for. Conversely, if the fibril stiffness is much smaller than the bulk stiffness, the energy storage in the fibril will dominate the work-of-separation and this mechanism is virtually unaffected by the fibril width.

\section{Sequential fibril failure}

In this section, the results for sequential failure of the fibrillar interface (schematically shown in Fig. 3b) are presented. First, the results for mode I sequential failure are presented and compared to the results for the simultaneous mode I failure case. The influence of the loading angle on the results is analyzed thereafter.

\subsection{Mode I loading}

Figs. 7a,b show an example of the deformed geometry of a mode I sequential failure analysis on a fibrillar interface in which the colors indicate the SED. It can be observed that material is drawn to the peel front from the adjacent bulk material. Upon reaching the fibril failure criterion (Fig. 7a), the fibril is released (Fig. 7b). The change in strain energy density between these two deformation states illustrates the energy dissipation for one fibril failure event, as depicted in Fig. 7c in which the calculated values are projected on the geometry prior to failure. Interestingly, the energy loss predominantly takes place in the bulk layer surrounding the failing fibril (indicated by 'SED decrease' in the figure), while the strain energy in the next fibril increases ('SED increase' in the figure). Note that, in spite of the mode I loading, the fibrils are inclined due to the asymmetry of the delamination front.

Fig. 8a shows the calculated work-of-separation resulting from the fibril energy loss for various fibril widths. Comparing Fig. 6a and Fig. 8a shows that both the sequential mode I and simultaneous failure 
cases result in almost identical results for the fibril energy loss. Apparently, the individual fibrils are predominantly loaded in tension upon failure for both loading cases. Previously reported results (Vossen et al., 2014, 2015) revealed that the energy loss in the fibrils is not the main dissipative mechanism for the investigated $\mathrm{Cu}$-PDMS system. Furthermore, it appears that the energy loss in the fibrils can be predicted analytically for the considered fibrillar structures. Therefore, in the remainder of this paper we will only focus on the bulk energy loss resulting from the discrete loading by the fibrils.

Fig. $8 \mathrm{~b}$ shows the bulk energy loss which, similar to Section 3.1.2, scales with the fibril width. The slope of the curve approximately equals 1 , but the curve differs from simultaneous fibril failure as no lower bound plateau (corresponding to a uniformly deformed bulk layer) is present for small $w$. This suggests that the energy stored in the bulk layer further away from the fibrils, is not released in an unstable manner, but instead recovered by the load bearing part of the structure in a stable manner, and therefore not contributing to the work-of-separation.

By comparing Fig. 6b and Fig. 8b, it is clear that for large $w$, sequential fibril failure leads to an increase in the bulk energy loss by approximately a factor of 4 compared to simultaneous fibril failure. This implies that the spatial discreteness of the fibrils and the resulting interaction between the fibrils is essential to explain the high work-of-separation values. This also suggests that continuum-based cohesive zone elements cannot be trivially used to model such an interfacial system, since the cohesive zone elements are not able to properly account for the spatial discreteness of the fibrils. Moreover, in the limit towards continuously distributed fibrils $(w \rightarrow 0$ for $w=h$ ), the bulk work-of-separation vanishes. Accordingly, (continuous) cohesive zones applied at the macroscopic scale would have to repair for this by assigning the work-of-separation to the interface, even though this is physically incorrect.
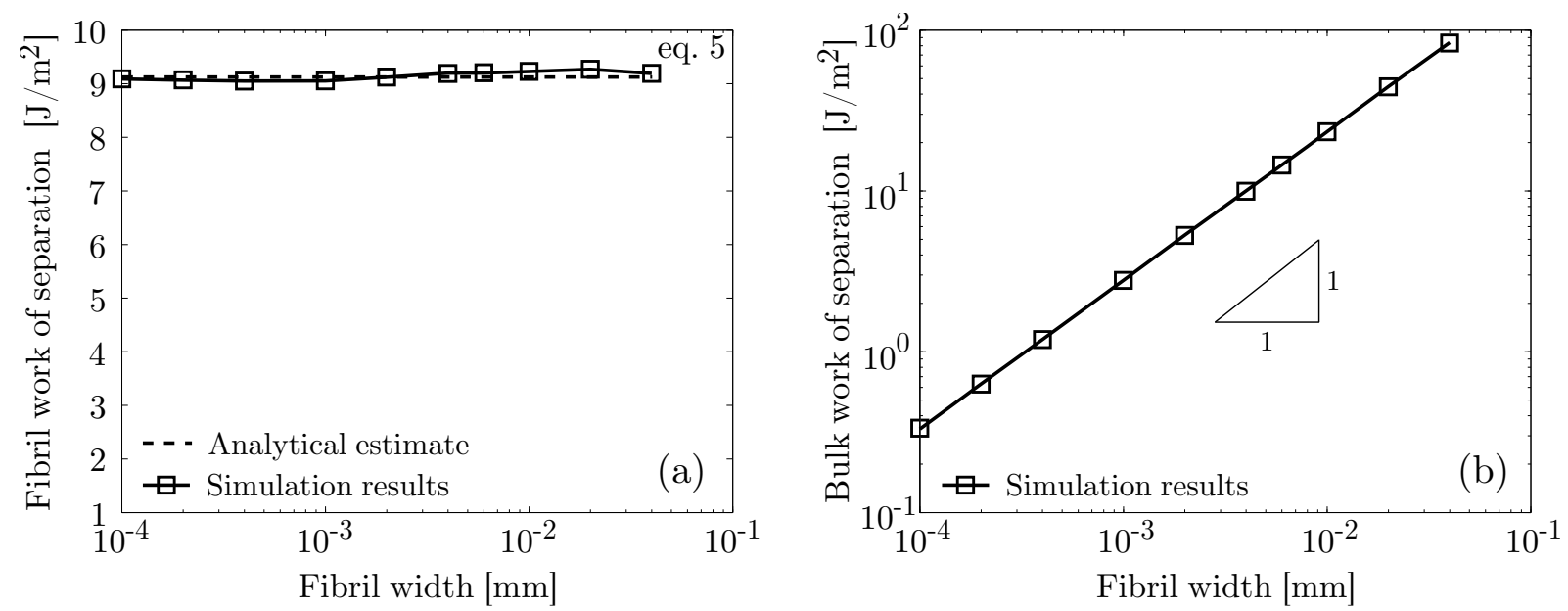

Figure 8: Work-of-separation for mode I sequential fibril failure from (a) the fibril and (b) the bulk. The dashed line represents the analytical estimate, the solid lines are the numerical results, where the squares indicate the data points. 

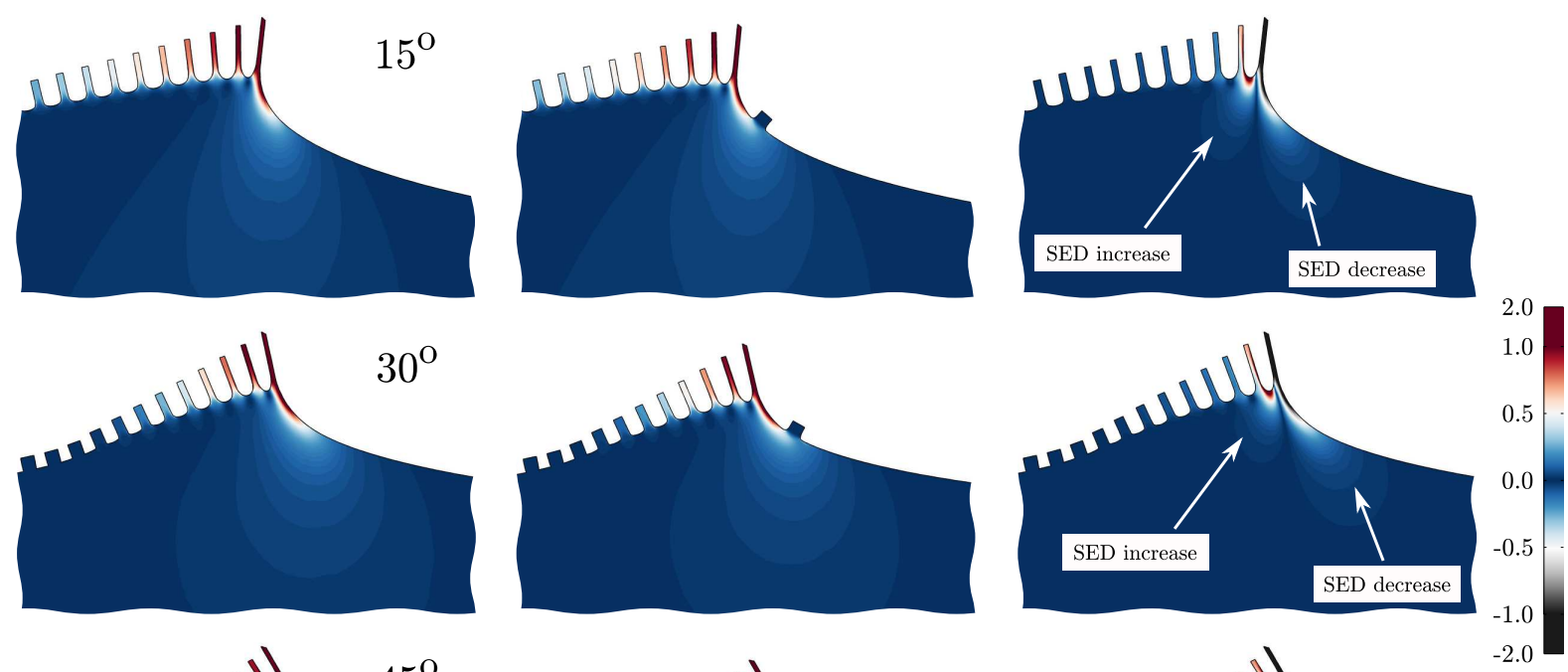

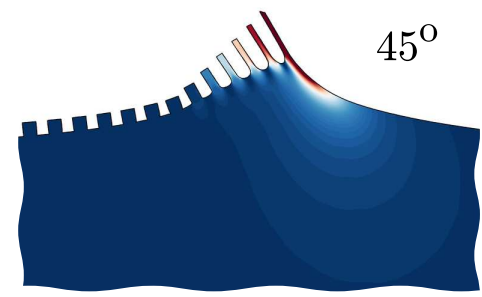

(a) Before failure

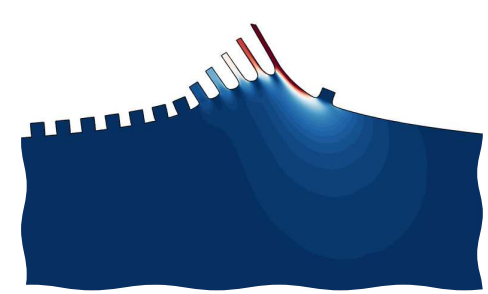

(b) After failure

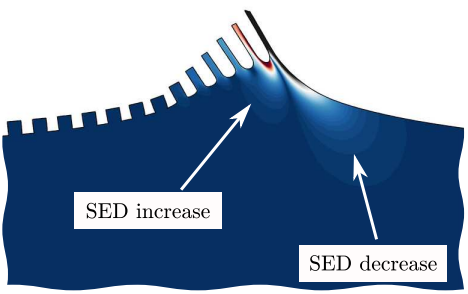

(c) SED change

Figure 9: Influence of the loading angle on the delamination mechanics, $w=0.01 \mathrm{~mm}$. The colors indicate the strain energy density $\left[\mathrm{N} / \mathrm{mm}^{2}\right]$. (a) The fibril reaches the failure criterion. (b) The geometry after instantaneous failure of the critical fibril. (c) SED after failure minus the SED before failure, projected on the geometry before failure.

\subsection{Mixed-mode loading}

Evidently, peel tests can be performed under various loading angles. For example, Neggers et al. (2015) performed both $0^{\circ}$ and $90^{\circ}$ peel tests on (fibrillating) Cu-PDMS interfaces. Therefore, the influence of the loading angle on the work-of-separation is studied for $w=0.01 \mathrm{~mm}$. Fig. 9 shows the deformed geometry for several loading angles. It can be observed that for all loading angles, a volume of bulk material is highly deformed near the critical fibril.

From these simulations, the bulk contribution to the work-of-separation is determined, see Fig. 10. No significant influence of the loading angle is visible which may seem surprising, as mode dependency is a commonly reported phenomenon for interface fracture toughness values (Hutchinson and Suo, 1992; Reeder and Crews, 1990). However, peel tests on these particular metal-elastomer fibrillating interfaces reveal only a negligible mode dependency (Neggers et al., 2015). These numerical results thus provide a physical explanation for the experimental observations. For the different loading angles, the bulk volume adjacent to the failing fibril is relatively free to deform. As the load exerted by the fibril on the bulk is equal for all loading angles, the amount of energy stored and released in an unstable manner in the bulk is not significantly affected by the loading angle. Indeed, Fig. 9c shows that the volume in which the majority of the energy loss occurs is approximately equal for the different loading angles. On the other hand, the loading state of the fibrils further away from the delamination front is different for different 


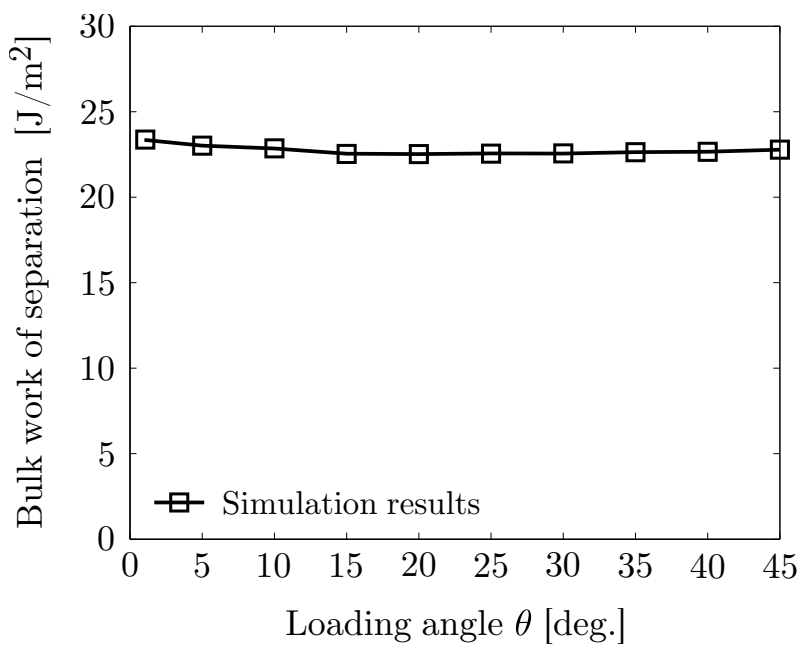

Figure 10: The bulk work-of-separation is not significantly influenced by the loading angle.

loading angles, but at this stage, this mainly concerns stored energy only. Since no pronounced effect of the loading angle on the work-of-separation is observed, the unstable bulk energy release indeed mainly occurs in the vicinity of the failing fibril, and not in the material further away.

\section{Influence of the failure stress}

To study the influence of the critical stress, a loading angle of $45^{\circ}$ is used. This value originates from observations in earlier work, where it was shown that the peel force in a $90^{\circ}$ peel test is actually transferred through the interface at an angle of approximately $45^{\circ}$ (Neggers et al., 2015). The critical stress is varied between $p_{c}=0.5 \mathrm{MPa}$ and $p_{c}=4 \mathrm{MPa}$, and $w=0.01 \mathrm{~mm}$. The resulting deformed geometries are shown in Fig. 11. Comparison of the calculated peel front geometries with the experimentally measured shapes (Neggers et al., 2015) confirm the appropriateness of the proposed set of boundary conditions. In addition, it can be observed that for $p_{c}=0.5 \mathrm{MPa}$, hardly any lift-off geometry is present, while increasing the critical stress leads to an increase in the peel front height, i.e., the fracture process zone increases.

Fig. 12a shows the bulk work-of-separation as a function of the critical stress. The significant effect of the critical stress on the work-of-separation can be clearly recognized. It can be observed that the slope of the curve slightly decreases with increasing critical stress. This is caused by the hardening in the rubber material response at large deformations. For sufficiently high values of $p_{c}$ the material response is highly nonlinear and consequently, only a slight increase in deformation (accompanied by a small increase in energy storage) is required to achieve considerably higher stress levels.

The peel front height (as defined in the inset of the figure) versus the critical stress is shown in Fig. 12b. The shape of the curve is similar to that of the work-of-separation, and therefore suggests a correlation between the work-of-separation and the peel front height. 

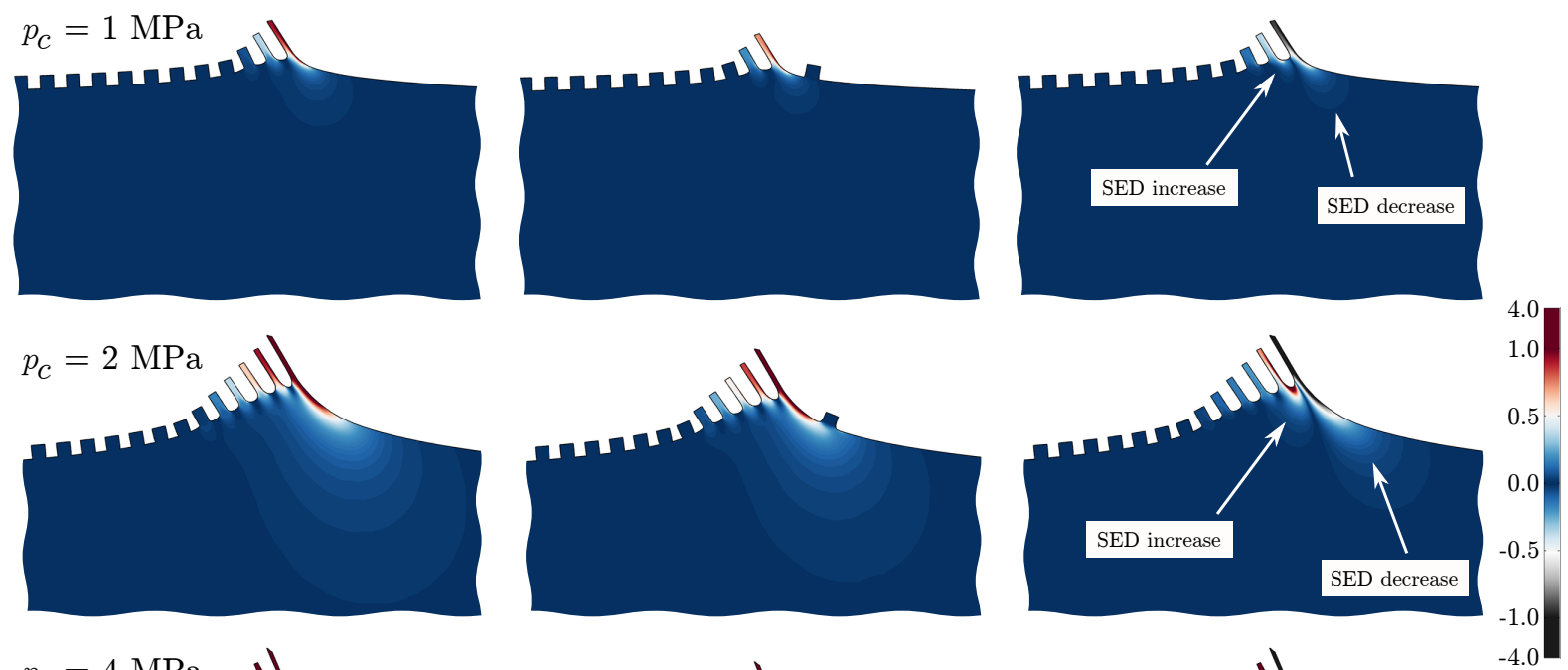

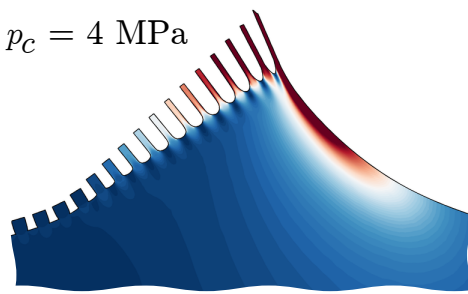

(a) Before failure

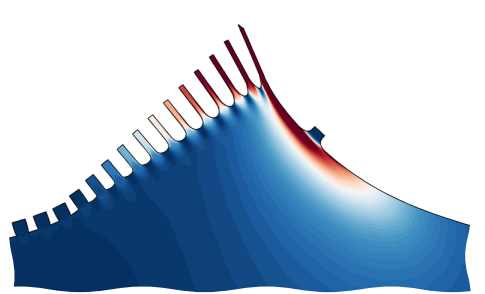

(b) After failure

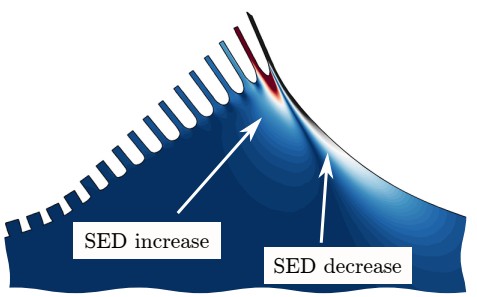

(c) SED change

Figure 11: Influence of the critical stress $p_{c}$ on the delamination mechanics, $w=0.01$ mm. The colors indicate the strain energy density $\left[\mathrm{N} / \mathrm{mm}^{2}\right]$. (a) The fibril reaches the failure criterion. (b) The geometry after instantaneous failure of the critical fibril. (c) SED after failure minus the SED before failure, projected on the geometry before failure.
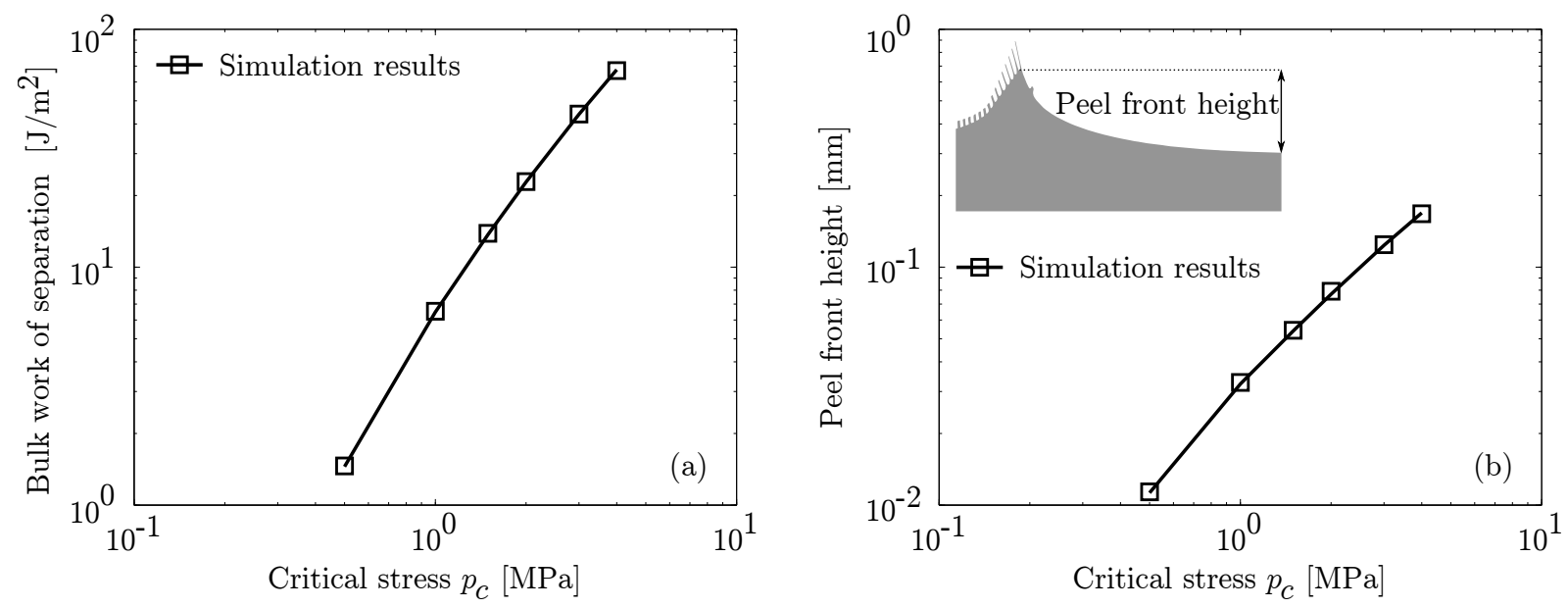

Figure 12: Influence of the critical stress $p_{c}$ on (a) the bulk work-of-separation and (b) the peel front height. 


\section{On the relation between the work-of-separation and the peel front height}

The results presented in the preceding section clearly indicate a relation between the macroscopic work-of-separation and the peel front height. In order to establish this relation, several fibril width values are considered. For each fibril width, in analogy with the preceding section, the critical stress is varied between $p_{c}=0.5 \mathrm{MPa}$ and $p_{c}=4 \mathrm{MPa}$. Fig. 13 depicts the work-of-separation as a function of the peel front height, for all considered fibril widths. Remarkably, the curves almost coincide. Although not shown in the figure, similar curves have been obtained for other $h / w$-ratios, which implies that the obtained relation between the work-of-separation and the peel front height is valid not only for the specific case $w=h$. These results confirm that the peel front height, emanating from the discrete nature of the fibrils, can be used to predict the work-of-separation.

In order to confront the predicted values to data from previously reported experimental results, the values obtained from $90^{\circ}$ peel tests of the same PDMS grade are indicated by the circles in Fig. 13 . Neggers et al. (2015) reported a work-of-separation of $100 \mathrm{~J} / \mathrm{m}^{2}$ at a peel front height of approximately $0.2 \mathrm{~mm}$, although they used a PDMS layer with a height of $0.75 \mathrm{~mm}$ instead of $1 \mathrm{~mm}$. For this peel front height, without knowledge of the exact fibrillar microstructure, the discrete fibrils model data used here suggests a work-of-separation of $89 \mathrm{~J} / \mathrm{m}^{2}$, which is reasonably close to the experimental value, especially considering the absence of the fibril contribution in the model result. Using a different substrate with a roughened surface, a work-of-separation of $300 \mathrm{~J} / \mathrm{m}^{2}$ was measured, at a peel front height of approximately $0.5 \mathrm{~mm}$. Extrapolation of the present results leads to $356 \mathrm{~J} / \mathrm{m}^{2}$ for this case. Finally, using a PDMS layer with the same height as used here, Van der Sluis et al. (2011) reported $1343 \pm 51 \mathrm{~J} / \mathrm{m}^{2}$ at a peel front height of $1.31 \pm 0.02 \mathrm{~mm}$. The extrapolation used here suggests a value of $1499 \mathrm{~J} / \mathrm{m}^{2}$, which is again a good quantitative approximation of the experimentally measured value. It appears that the predicted workof-separation values from the peel front height are in good agreement with the experimentally measured values. It can thus be concluded that the identified mechanisms in the discrete fibrils model provide a proper mechanistic explanation for the high work-of-separation values of fibrillating metal-elastomer interfaces.

\section{Conclusions}

The huge gap that exists between the low adhesion energy at microscopic scale and the high experimentally measured macroscopic work-of-separation in fibrillating metal-elastomer systems was analyzed in this paper. Earlier work based on the formation and deformation of a single fibril up to failure provided a significantly larger work-of-separation value than the small-scale interface adhesion, yet a decade too small with respect to the experimental values. In this contribution, a systematic study was performed by 


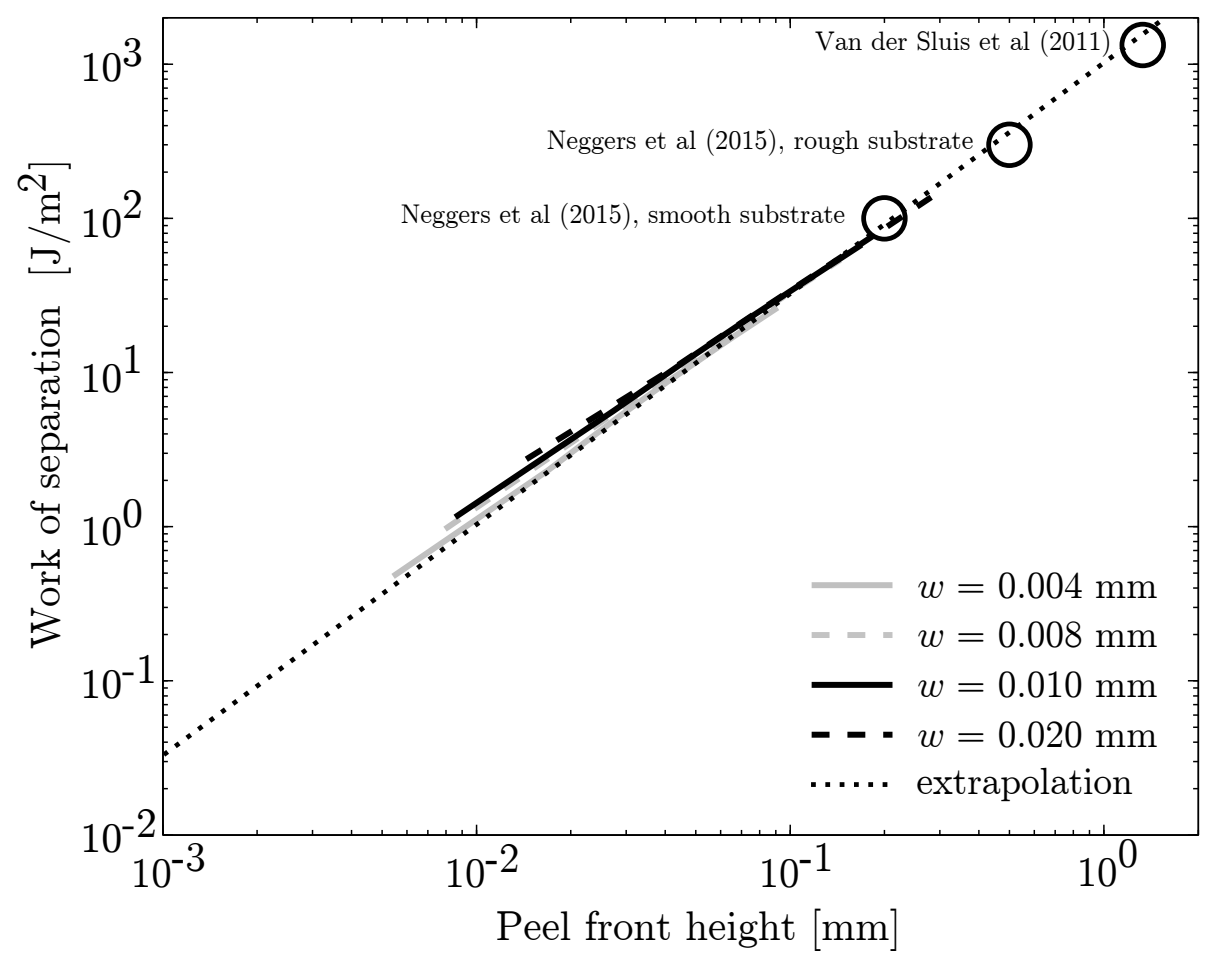

Figure 13: Work-of-separation versus the peel front height, as a result of the discrete fibrils, for several fibril widths. Extrapolation of the results indicates a good match with the experimental data obtained for $90^{\circ}$ peel tests of the same PDMS grade, indicated by the circles.

considering multiple discrete hyperelastic fibrils within the fracture process zone. As a result, the high work-of-separation values could be explained by the following phenomena:

- the dynamic release of the stored elastic energy by fibril fracture;

- the spatial discreteness of multiple fibrils as well as the interaction of these fibrils with the adjacent deforming bulk elastomer material;

- the highly nonlinear behavior of the elastomer.

In addition, the results established a quantitative relation between the peel front height (i.e. the fracture process zone), emanating from the discrete fibrils, and the macroscopic work-of-separation, and was validated with experimental results. The identified dissipative mechanism was not reported before and contrasts the results established for pressure sensitive adhesives, where fibril elongation, and the dissipation that goes along with it, has been known to be the dominant contribution to the work-ofseparation.

Finally, in the case of sequential fibril failure, it was shown that the bulk work-of-separation vanished when approaching the continuum limit (i.e. $w \rightarrow 0$ ). This illustrates a shortcoming of cohesive zone models which have to repair for this by assigning the work-of-separation to the interface, even though this is physically not the case. 


\section{Acknowledgements}

This research is supported by the Dutch Technology Foundation STW, which is part of the Netherlands Organisation for Scientific Research (NWO), and which is partly funded by the Ministry of Economic Affairs under Project Nr. 10108.

\section{References}

Brown, K., Hooker, J.C., Creton, C., 2002. Micromechanisms of tack of soft adhesives based on styrenic block copolymers. Macromolecular Materials and Engineering 287, 163-179.

Coosemans, J., Hermans, B., Puers, R., 2006. Integrating wireless ECG monitoring in textiles. Sensors and Actuators, A: Physical 130-131, 48-53.

Creton, C., 2003. Pressure-sensitive adhesives: An introductory course. MRS Bulletin 28, 434-439.

Glassmaker, N.J., Himeno, T., Hui, C.Y., Kim, J., 2004. Design of biomimetic fibrillar interfaces: 1. Making contact. Journal of the Royal Society Interface 1, 23-33.

Glassmaker, N.J., Jagota, A., Hui, C.Y., 2005. Adhesion enhancement in a biomimetic fibrillar interface. Acta Biomaterialia 1, 367-375.

Gonzalez, M., Axisa, F., Bulcke, M.V., Brosteaux, D., Vandevelde, B., Vanfleteren, J., 2008. Design of metal interconnects for stretchable electronic circuits. Microelectronics Reliability 48, 825-832.

Guidoni, G.M., Schillo, D., Hangen, U., Castellanos, G., Arzt, E., McMeeking, R.M., Bennewitz, R., 2010. Discrete contact mechanics of a fibrillar surface with backing layer interactions. Journal of the Mechanics and Physics of Solids 58, 1571-1581.

Hoefnagels, J.P.M., Neggers, J., Timmermans, P.H.M., Van der Sluis, O., Geers, M.G.D., 2010. Copperrubber interface delamination in stretchable electronics. Scripta Materialia 63, 875-878.

Hsu, Y.Y., Gonzalez, M., Bossuyt, F., Axisa, F., Vanfleteren, J., De Wolf, I., 2009. In-situ observation on deformation behavior and stretching-induced failure of fine pitch stretchable interconnect. Journal of Materials Research 24, 3573-3582.

Hsu, Y.Y., Gonzalez, M., Bossuyt, F., Axisa, F., Vanfleteren, J., De Wolf, I., 2010. The effect of pitch on deformation behavior and the stretching-induced failure of a polymer-encapsulated stretchable circuit. Journal of Micromechanics and Microengineering 20, 075036.

Hui, C.Y., Glassmaker, N.J., Jagota, A., 2005. How compliance compensates for surface roughness in fibrillar adhesion. The Journal of Adhesion 81, 699-721. 
Hui, C.Y., Glassmaker, N.J., Tang, T., Jagota, A., 2004. Design of biomimetic fibrillar interfaces: 2. mechanics of enhanced adhesion. Journal of the Royal Society Interface 1, 35-48.

Hui, C.Y., Jagota, A., Lin, Y.Y., Kramer, E.J., 2002. Constraints on microcontact printing imposed by stamp deformation. Langmuir 18, 1394-1407.

Hui, C.Y., Jagota, A., Shen, L., Rajan, A., Glassmaker, N., Tang, T., 2007. Design of bio-inspired fibrillar interfaces for contact and adhesion - theory and experiments. Journal of Adhesion Science and Technology 21, 1259-1280.

Hutchinson, J.W., Suo, Z., 1992. Mixed mode cracking in layered materials. Advances in Applied Mechanics 29, 63-191.

Jagota, A., Bennison, S.J., 2002. Mechanics of adhesion through a fibrillar microstructure. Integrative and Comparative Biology 42, 1140-1145.

Jagota, A., Hui, C.Y., 2011. Adhesion, friction, and compliance of bio-mimetic and bio-inspired structured interfaces. Materials Science and Engineering R: Reports 72, 253-292.

Khang, D.Y., Jiang, H., Huang, Y., Rogers, J.A., 2006. A stretchable form of single-crystal silicon for high-performance electronics on rubber substrates. Science 311, 208-212.

Kim, D.H., Lu, N., Ghaffari, R., Kim, Y.S., Lee, S.P., Xu, L., Wu, J., Kim, R.H., Song, J., Liu, Z., Viventi, J., De Graff, B., Elolampi, B., Mansour, M., Slepian, M.J., Hwang, S., Moss, J.D., Won, S.M., Huang, Y., Litt, B., Rogers, J.A., 2011a. Materials for multifunctional balloon catheters with capabilities in cardiac electrophysiological mapping and ablation therapy. Nature Materials 10, 316-323.

Kim, D.H., Lu, N., Ma, R., Kim, Y.S., Kim, R.H., Wang, S., Wu, J., Won, S.M., Tao, H., Islam, A., Yu, K.J., Kim, T.I., Chowdhury, R., Ying, M., Xu, L., Li, M., Chung, H.J., Keum, H., McCormick, M., Liu, P., Zhang, Y.W., Omenetto, F.G., Huang, Y., Coleman, T., Rogers, J.A., 2011b. Epidermal electronics. Science 333, 838-843.

Krishnan, V.R., Hui, C.Y., 2008. Large deformation of soft elastic materials in adhesive contact with a rigid cylindrical flat punch. Soft Matter 4, 1909-1915.

Lakrout, H., Sergot, P., Creton, C., 1999. Direct observation of cavitation and fibrillation in a probe tack experiment on model acrylic pressure-sensitive-adhesives. Journal of Adhesion 69, 307-359.

Li, T., Suo, Z., 2007. Ductility of thin metal films on polymer substrates modulated by interfacial adhesion. International Journal of Solids and Structures 44, 1696-1705. 
Li, T., Suo, Z., Lacour, S.P., Wagner, S., 2005. Compliant thin film patterns of stiff materials as platforms for stretchable electronics. Journal of Materials Research 20, 3274-3277.

Long, R., Hui, C.Y., Kim, S., Sitti, M., 2008. Modeling the soft backing layer thickness effect on adhesion of elastic microfiber arrays. Journal of Applied Physics 104.

Lu, N., Wang, X., Suo, Z., Vlassak, J., 2007. Metal films on polymer substrates stretched beyond 50\%. Applied Physics Letters 91.

Maas, S.A., Ellis, B.J., Ateshian, G.A., Weiss, J.A., 2012. FEBio: Finite elements for biomechanics. Journal of Biomechanical Engineering 134.

Neggers, J., Hoefnagels, J., van der Sluis, O., Sedaghat, O., Geers, M., 2015. Analysis of the dissipative mechanisms in metal-elastomer interfaces. Engineering Fracture Mechanics 149, 412-424.

Noderer, W.L., Shen, L., Vajpayee, S., Glassmaker, N.J., Jagota, A., Hui, C.Y., 2007. Enhanced adhesion and compliance of film-terminated fibrillar surfaces. Proceedings of the Royal Society of London A: Mathematical, Physical and Engineering Sciences 463, 2631-2654.

Persson, B.N.J., 2003. On the mechanism of adhesion in biological systems. Journal of Chemical Physics $118,7614-7621$.

Porwal, P.K., Hui, C.Y., 2008. Strength statistics of adhesive contact between a fibrillar structure and a rough substrate. Journal of the Royal Society Interface 5, 441-448.

Reeder, J.R., Crews, J.R., 1990. Mixed mode bending method for delamination testing. AiAA Journal $28,1270-1276$.

Rogers, J.A., Someya, T., Huang, Y., 2010. Materials and mechanics for stretchable electronics. Science 327, 1603-1607.

Shen, S., Soh, A.K., 2008. Tuning the geometrical parameters of biomimetic fibrillar structures to enhance adhesion. Journal of the Royal Society Interface 5, 373-382.

Shull, K.R., Creton, C., 2004. Deformation behavior of thin, compliant layers under tensile loading conditions. Journal of Polymer Science, Part B: Polymer Physics 42, 4023-4043.

Sitti, M., Fearing, R.S., 2003. Synthetic gecko foot-hair micro/nano-structures as dry adhesives. Journal of Adhesion Science and Technology 17, 1055-1073.

Van der Sluis, O., Hsu, Y.Y., Timmermans, P.H.M., Gonzalez, M., Hoefnagels, J.P.M., 2011. Stretchinginduced interconnect delamination in stretchable electronic circuits. Journal of Physics D: Applied Physics 44, 034008. 
Song, J., Jiang, H., Liu, Z.J., Khang, D.Y., Huang, Y., Rogers, J.A., Lu, C., Koh, C.G., 2008. Buckling of a stiff thin film on a compliant substrate in large deformation. International Journal of Solids and Structures 45, 3107-3121.

Tang, T., Hui, C.Y., Glassmaker, N.J., 2005. Can a fibrillar interface be stronger and tougher than a non-fibrillar one? Journal of the Royal Society Interface 2, 505-516.

Vossen, B.G., Schreurs, P.J.G., Van der Sluis, O., Geers, M.G.D., 2014. Multi-scale modeling of delamination through fibrillation. Journal of the Mechanics and Physics of Solids 66, 117-132.

Vossen, B.G., Van der Sluis, O., Schreurs, P.J.G., Geers, M.G.D., 2015. On the role of fibril mechanics in the work of separation of fibrillating interfaces. Mechanics of Materials $88,1-11$.

Yuk, H., Zhang, T., Lin, S., Parada, G., Zhao, X., 2016. Tough bonding of hydrogels to diverse non-porous surfaces. Nature Materials 15, 190-196.

Zhao, X., 2014. Multi-scale multi-mechanism design of tough hydrogels: building dissipation into stretchy networks. Soft Matter 10, 672-687.

Zosel, A., 1998. The effect of fibrilation on the tack of pressure sensitive adhesives. International Journal of Adhesion and Adhesives 18, 265-271. 